\title{
Semantic Domains in Akkadian Texts
}

\author{
Saana Svärd, Heidi Jauhiainen, Aleksi Sahala, and Krister Lindén
}

\section{Introduction $^{1}$}

The Mesopotamian civilizations have left behind rich textual material in the Akkadian language, written in cuneiform script on artifacts excavated from that area. ${ }^{2}$ A variety of cuneiform scripts were used to write different languages in the ancient Near East, ${ }^{3}$ but we are here interested in what has often been called the Mesopotamian cuneiform tradition, which means texts mostly written in Sumerian (which has no known language relatives) and Akkadian (a Semitic language). Michael Streck estimated in 2010 that the number of published texts in Sumerian was approximately 102,300 (3.076 million words) and in Akkadian was approximately 144,000 ( 9.9 million words) ${ }^{4}$ Of course, the published texts are only a segment of the total number of objects with cuneiform text in the museums of the world, and there are doubtless still more texts that have not been excavated that are still unknown to us. Due to the challenges of the cuneiform script, as well as the small number of Assyriologists, the texts are being published at a slow rate, but the number of published texts is continuously growing. According to some estimates, only 10 percent of the excavated texts on cuneiform tablets have been published. Since the late 1990s,

1 We thank Dr. Sebastian Fink for his feedback and Dr. Albion Butters for helping to improve the English of this essay. We also gratefully recognize the financial support of the Academy of Finland for the writing of this essay.

2 Mesopotamia refers to a region situated within the Tigris-Euphrates river system, in modern days roughly corresponding to most of Iraq and the eastern parts of Syria. A number of cultures and political units flourished in the area. The most prominent political entities that used Akkadian are usually referred to as Assyria and Babylonia.

3 The cuneiform script was originally developed by Sumerians c. 3000 BCE. In the following centuries, the script was adapted for Akkadian (a Semitic language in use from about 2500 to 500 BCE). It was also used to write several other ancient Near East languages, such as Hittite and Elamite. Although passing centuries and new languages caused the form of the signs to change, the basic shapes - triangular wedges left by a reed stylus—stayed the same.

4 Streck 2010; see esp. the summary on pages $53-54$. Here "published text" refers to a cuneiform text that has been made available as a drawing, transliteration, or translation in a credible scholarly publication.

(C) SAANA SVÄRD ET AL., 2018 | DOI 10.1163/9789004375086_009

This is an open access chapter distributed under the terms of the prevailing CC-BY-NC License. 
Assyriology has made significant advances in making the texts available in electronic corpora, although it is still not self-evident that all published texts should be made electronically available. The aim of this contribution is to examine two possible language-technology methodologies for analyzing semantic fields in Akkadian. ${ }^{5}$ Our research group used the existing electronic Open Richly Annotated Cuneiform Corpus (Oracc) ${ }^{6}$ Oracc is one of the largest corpora of Sumerian and Akkadian texts, consisting of over seventeen thousand texts (almost two million words). ${ }^{7}$ Roughly half of it has been annotated. Annotation and metadata are crucially important, ${ }^{8}$ since not even a well-trained Assyriologist finds transliterated Akkadian as easy to read as a newspaper. The script uses both syllabic and logographic signs, and each sign can be read in many different ways. ${ }^{9}$ Akkadian also uses complex inflection; that is, words are modified in order to express various grammatical categories. Furthermore, the precise genre, time period, and expected vocabulary and orthography need to be taken into account. In addition to more traditional Assyriological methods, this project explores two language-technological methods: Pointwise Mutual Information (PMI) and Word2vec.

In the second section of this chapter, we discuss the background for the research and our source material. The third section outlines the theoretical framework underlying our work and how that framework connects with language technology. The fourth section presents hypothetical semantic fields for our test lexemes "horse," "to speak," and "power," based on Assyriological research. These semantic fields will act as a hypothetical baseline against which the results of our chosen language-technological methods may be compared. The fifth section describes the PMI method and reports the results of our test lexemes. The sixth section describes the Word2vec method and reports on the

5 Language Technology is a multidisciplinary field that studies and develops methods for processing human language with the help of computers.

6 The research group's work consists of two projects: "Semantic Domains in Akkadian texts" (principal investigator: Krister Lindén) and "Deep Learning and Semantic Domains in Akkadian Texts" (principal investigator: Saana Svärd). Oracc: <http://oracc.museum.upenn. edu/> (accessed June 30, 2017). We would like to thank Professor Niek Veldhuis (University of California, Berkeley) for his assistance with Oracc. For further information, see in this volume, Pagé-Perron, 198-200.

7 Our numbers are from September 2017.

8 In corpus linguistics, an annotation is a comment that specifies the various linguistic features of a word. Metadata provides additional information about the text in question. In Assyriology, this information includes date and provenance.

9 A syllabic sign represents a syllable, and a logographic sign represents a word. 
results achieved with it so far. The concluding section discusses the results and outlines some paths forward.

\section{Theoretical and Methodological Background and Source Material}

In this section, we will first highlight the general aims of our research group, setting out the larger framework for our whole endeavor. We conclude the section with a more detailed discussion of our source material and the specific aim of this project: to examine two language-technological methodologies for analyzing semantic fields in Akkadian.

The theoretical basis of our research lies in the well-tested and well-founded approach that research needs to consider the meaning of concepts from more than just an outsider's analytical perspective, but also from inside the group that is being studied. This principle of emic research underlies, for example, many anthropological studies of human culture. ${ }^{10}$ In general, concepts that are foreign to the culture that is being researched do not necessarily have much explanatory power. However, applying an emic approach is challenging in the case of the distant Mesopotamian civilizations, which are known through their texts, mostly written in Akkadian and Sumerian, as well as through archaeological finds from the region. We aim to answer this challenge through language itself.

Semantic research in Assyriology has very much consisted of qualitative research on individual concepts - that is, research done on a small amount of data without mathematical methods. Our research group, however, aims to do quantitative research — that is, to use statistical and computational techniques employed in the natural sciences on large amounts of Mesopotamian textual data. We seek to use state-of-the-art technology to handle the data in order to gain new semantic insights into ancient texts and cultures. Without the use of automated methods, this kind of research is extremely slow and often possible for only the few senior Assyriologists who have read through and absorbed thousands of Akkadian texts. Offering a quantitative perspective will broaden the possibilities of semantic and linguistic research on these text corpora by linguists and historians who have only a basic knowledge of the language. Overall, we aim to generate semantic domains for Akkadian lexemes using methods from language technology.

Word sense induction (also called word sense discrimination or word sense discovery) is the task of determining what meaning a word may have in

10 See, for example, Eriksen (2010, 39-40). 
different contexts. In some ways, the manual way of doing this has been one of the core concerns of Assyriology ever since scholars started to decipher the first cuneiform texts in the 1850s. However, no determined effort has been made to fully utilize the existing electronic corpora and language-technological approaches. Therefore, dependency parsers or other sophisticated automated tools are not available to create pre-analyzed contexts for words. Starting from scratch, our project plans to apply a number of different approaches to the corpus.

In general, language-technology-related research has rarely been pursued in Assyriology. For the Sumerian language, there are some studies, perhaps because Sumerian material has been available in useful formats longer than Akkadian. Stephen Tyndall used Naive Bayes and Maximum Entropy classifiers to reunite different fragments of the same text in Hittite. ${ }^{11}$ In Akkadian, a social network analysis approach has been used as an analytical tool, but not automatically. ${ }^{12}$ One previous, older work relating to Akkadian comes from Helsinki, where Laura Kataja and Kimmo Koskenniemi experimented on using computational morphology for Akkadian texts. ${ }^{13}$ Recently, Terhi NurmikkoFuller has been analyzing narrative structures in Sumerian literature from the point of view of the Semantic Web. ${ }^{14}$

Our data comes from the electronic text corpus Oracc. The corpus is regularly updated, but our data for this paper is a snapshot of the corpus from October 2016. ${ }^{15}$ Akkadian uses inflection; that is, words are modified in order to express various grammatical categories: for example, šarrum, "king," for the singular nominative form of the noun and sarram for the singular accusative form. This is why we decided to use only the lemmata - that is, the dictionary or citation forms - of various words when looking for semantic domains. Not all of our texts have been annotated with these dictionary forms, and most texts also contain unannotated words; therefore, we created a file containing all the texts where at least 10 percent of the words have been annotated with a dictionary form such as šarru, "king"; or kakku, "weapon." In general, in the

11 Tyndall 2012. Hittite was an Indo-European language of Anatolia (Asia Minor) written with cuneiform signs.

12 Waerzeggers, forthcoming.

13 Kataja and Koskenniemi 1988.

14 Nurmikko-Fuller 2016. The Semantic Web provides a common framework that allows data to be shared and reused across applications, enterprises, and community boundaries. For more information, see "W3C Semantic Web Activity," updated December 11, 2013, <https://www.w3.org/2001/sw/> (accessed June 21, 2017). 
Oracc corpus, the metadata added to the texts has been done during different projects over a number of years. This is why some texts have more metadata than others. Some texts include information on provenance, the period when they were written, and genre. Individual words may have such tags as transcription, dictionary form, translation, part of speech, and language. For example, a well-annotated sample word in our material, \{GIŠ\}TUKUL.MEš-ia, includes the following: the base form in Akkadian (kakku), part of speech (noun), transcription (kakkiya), translation lemma ("stick"), translation sense (weapon), and language (Akkadian). Additional metadata for the whole text can include its provenance (the city of Uruk) and period (Neo-Assyrian). ${ }^{16}$

In our processing of Oracc, such words as numerals, personal names, and divine names, as well as place names, were grouped together under the lemmata "numeral," "person," "divinity," and "place," respectively. Broken-off words and missing dictionary forms were replaced with the underline character (" while partly broken words were left as they are in Oracc. Furthermore, the cuneiform script does not mark the end of a sentence, and this is not indicated in any way in our texts. Hence, each cuneiform document was considered as one line of text. When experimenting with the file produced this way, we noticed that various prepositions and adverbs were too prominently present in the results of the analysis. Hence, we modified the file by changing all words that had not been annotated as nouns, verbs, or adjectives into an underline character ("_"). All in all, this latter file contains 962,868 words from 8,392 different texts. We used the file for analysis with both methods of language technology chosen for this paper.

Regarding the genre of our texts, most of our material are either letters, historical texts, legal texts, or scholarly texts. Counted together, these genres form an overwhelming majority (more than seven thousand texts). Chronologically speaking, about two-thirds of our material (more than six thousand texts) is from the late Neo-Assyrian period or later (written roughly after 800 BCE). Finally, almost half of the texts come from Nineveh, and a significant portion (more than a thousand) are from Uruk. As expected, a large number of texts (approximately 1,500) have uncertain origins. These factors naturally influence our results.

In the context of language technology, distributional semantic models keep track of the appearances of words according to their proximity to each other in

16 For further examples, see the Korp interface at $<$ https://korp.csc.fi/?mode=other_lang uages\#?lang=en\&stats_reduce $=$ word\&cqp=[]\&corpus=oracc_dcclt\&search=word|lugal $>$ (accessed July 1, 2017). 
order to measure their similarity. ${ }^{17}$ These models can be computed by counting the frequency of all the words in relation to their neighboring words or, more recently, by predicting the context where words appear. The Pointwise Mutual Information (PMI ${ }^{18}$ method is a count model, whereas Word2vec uses socalled Artificial Neural Networks (ANNs) to predict word co-occurrence. ${ }^{19}$ In the current essay, we take the meanings of Akkadian words, as defined in the Chicago Assyrian Dictionary (CAD), as a departure point. ${ }^{20}$ The results gleaned from analyzing the $C A D$ will then act as a comparison point to the results gleaned by PMI and Word2vec from the file generated from the Oracc data. The following sections will explore in more detail how we analyze semantic fields in Akkadian with the help of PMI and Wordzvec.

\section{Emic Approach and Linguistic Departure Points}

As the aim of this section is to discuss our theoretical and methodological departure points, we start by indulging in some history of the field of Assyriology. The eminent Assyriologist Benno Landsberger emphasized the conceptual autonomy of Mesopotamian culture as early as 1926, arguing that Mesopotamian concepts need to be understood on their own terms. He suggested that if one is approaching "the alien mind from a fixed system of conceptual referents ... I could always only find again in my object what I already had within my own perspective."21 In some ways, we see this as a kind of precursor to the emic principle (see "Theoretical and Methodological Background and Source Material," above), which suggests that a culture needs to be understood on its own terms.

17 The field of distributional semantics encompasses the study of semantic similarities between linguistic items by using mathematical methods to see how they are spread throughout a large dataset. See, for example, Baroni, Dinu, and Kruszewski (2014). See also early work by John R. Firth.

18 Church and Hanks 1989.

19 Mikolov et al. 2013a; Mikolov, Yih, and Zweig 2013. Neural networks: a computational model inspired by the human brain where interconnected nodes (neurons) work in parallel to find out how to solve a problem by themselves when given an example. See in this volume, applied a) to landscape archaeology, Ramazzotti, 63-65; b) to objects, in particular cylinder seals, Ludovico, 92-94.

The $C A D$ (see in bibliography Roth 1956-2011) is the best and most extensive dictionary of Akkadian, although some parts of it are already fairly old, as the dictionary project took place from 1921 until 2011. The volumes are freely downloadable at $<$ https://oi.uchicago. edu/research/publications/assyrian-dictionary-oriental-institute-university-chicagocad> (accessed June 21, 2017), but they are not fully searchable.

21 This English translation appears in Landsberger $(1976,60)$, but the original idea was first published in 1926 . 
Anthropologists have engaged the same problem..$^{22}$ At the root of the dilemma is that Western models of thinking are far from universal. At the same time, while concepts that are foreign to the culture that is being researched can be used in research (the so-called etic approach), if the evidence is shoehorned into a modern mold, there is a danger of anachronistic explanations. As a simple example, taking marriage as a topic of study, defining marriage as a legal contract between men and women as a cornerstone of one's research is not very fruitful if one is researching a cultural system where there are more than two genders or where marriage does not have legal implications in the same sense as in the modern Western world.

A similar dilemma of emic vs. etic is well known in linguistics. In the twentieth century, formal linguists argued that individual languages were mere variations of the underlying universal cognitive language model. On the other hand, linguistic relativists opposed this and proved that different languages, as they are spoken by native speakers, do have real differences on the conceptual level. ${ }^{23}$ Linguistic relativity has a long and debated history, but here we concentrate on its connection to cognitive linguistics. ${ }^{24} \mathrm{~A}$ classic in that regard is George Lakoff's work, which argues against an "objectivist" view of categories reflecting reality, but suggests that categories are rooted in the body ("experimental realism") and have a lot to do with the physical and social environment. ${ }^{25}$ We subscribe to this view, which has been further explored in a field that is parallel to Assyriology: namely, the study of the Hebrew Bible.

In biblical studies, cognitive linguistics has been approached, for example, by James Barr and Ellen van Wolde. ${ }^{26}$ We found the work of Reinier de Blois to be especially useful; it argues strongly for the application of cognitive linguistics. According to Blois, "Where traditional linguistic theory claims that words have meanings, the cognitive linguist would say that meanings have words." ${ }^{27}$

Every word is a member of a larger group of words and shares certain aspects of meaning with them. From a paradigmatic perspective, people categorize concepts in paradigmatic cognitive categories, also referred to as lexical semantic domains. For example, in English, the concept "orange" belongs to the lexical semantic domain "fruit," together with pears and apples.

22 For one example, see Arens and Karp (1989).

23 Levinson 2003, 14-16; Fleisch 2007, 41-42, 46. See also Dirven and Verspoor (2004) and Geeraerts (2010).

24 See also in this volume, Bigot Juloux, 170-174, who uses cognitive linguistics for verbal categories.

25 Lakoff 1987.

26 Barr 1961; Wolde 2009.

27 Blois 2008b, 266. 


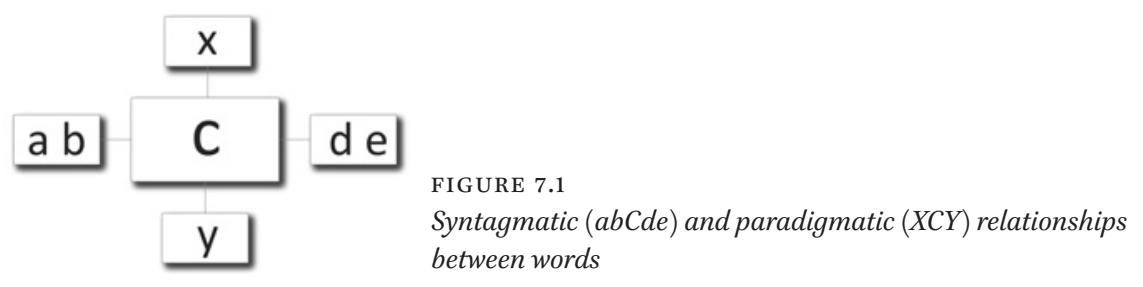

Simultaneously, concepts always associate with other concepts syntagmatically, forming contextual semantic domains. In other words, the lexeme "orange" can function "in different settings ... each of which evokes a different image in our minds." The HORTICULTURE-domain for "orange" evokes images of "tree," "ripe," "picking," etc., whereas the COMMERCE-domain for "orange" implies "booth," "seller," "money," etc. ${ }^{28}$

Thus, syntagmatic semantic domains are groups of words that "occur together in a prototypical scenario." ${ }^{29}$ We see the methods of language technology as the key to tracing such semantically relevant properties in large corpora and to providing Assyriologists with tools to reflect on the semantic domains of the words. The traditional Assyriological approach to building semantic fields is thus connected with the language-technological approaches by means of our focus on syntagmatic and paradigmatic relationships between words. In Figure 7.1 below, these relationships are arranged on two axes, abCde and $X c Y$. To give a concrete example of these relationships, Figure 7.2 shows the example relating to "orange" when it is situated in this diagram.

The following three methodological sections examine syntagmatic and paradigmatic relationships for three specific Akkadian lexemes: sisû, "horse"; qabû, "to speak"; and danānu, "to be strong, powerful." These lexemes were chosen because they are well attested in Akkadian and sufficiently distinct from each other. Another topic altogether is the connection of individual lexemes to more complex cultural concepts, but this question is not within the scope of the current paper. As we are, at the moment, only testing which language-technology methods might be useful for analyzing our data, in the first of the following three sections ("Traditional Examples of Semantic Fields") we use the traditional Assyriological method of examining words "by hand" with the literature of the field. In practice, we built a suggestion for both syntagmatic and paradigmatic fields with the help of the $C A D$. The two language-technological

28 Blois 2008b, 274 .

29 Blois 2008a, 206. 


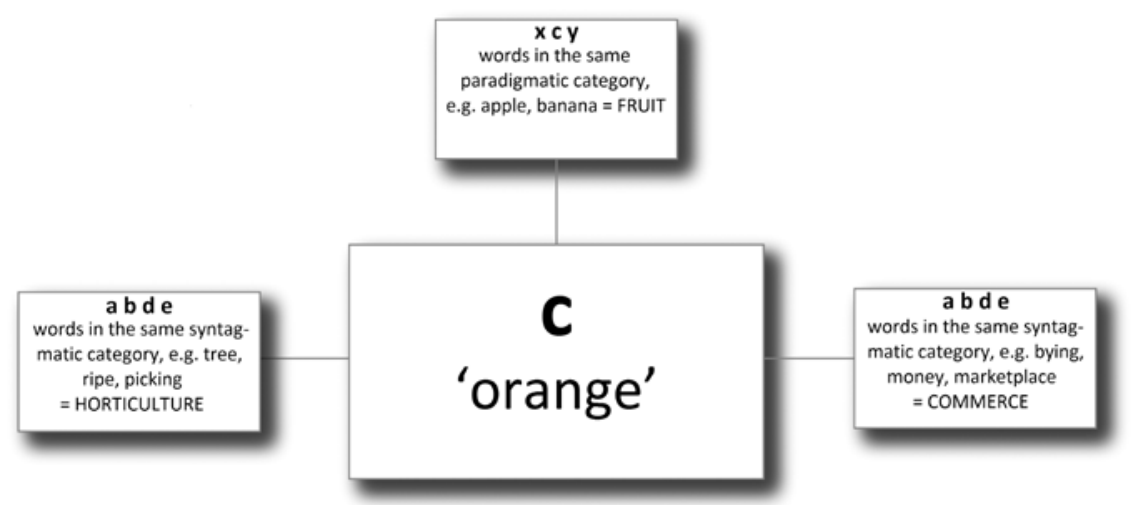

FIGURE 7.2 Syntagmatic and paradigmatic relationships, using "orange" as an example

methods which we chose to test here are PMI and Word2vec. PMI is a statistical method that finds the words that appear close to a target word, ${ }^{30}$ whereas Word2vec has been shown to capture the semantic relationships of words. ${ }^{31}$ These particular methods were chosen because PMI can be used to analyze the syntagmatic axis $a b C d e$, and Word2vec may be able to yield results regarding the paradigmatic $X C Y$ axis of our test words. The results of these analyses will be compared to the results of the Assyriological inquiry in "Pointwise Mutual Information" and "Word2vec." In other words, the results of the traditional method described in the following section will act as a kind of hypothesis in relation to which the results of PMI and Word2vec can be compared.

\section{Traditional Examples of Semantic Fields}

In the three subsections of this section, we outline both syntagmatic and paradigmatic relationships of three Akkadian lexemes: sis $\hat{u}$, "horse"; qabû, "to speak"; and danānu, "to be strong, powerful." Analysis of these relationships is based on existing Assyriological research, namely the $C A D .^{32}$ In practice, we

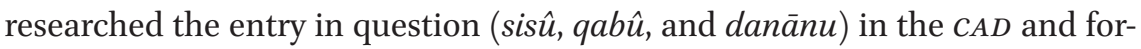
mulated a hypothetical syntagmatic and paradigmatic field based on the infor-

\footnotetext{
$30 \quad$ Church and Hanks 1989 .

$31 \quad$ Mikolov et al. 2013a; Mikolov, Yih, and Zweig 2013.

32 Although we use the $C A D$ as our source material, we follow here the writing conventions of A Concise Dictionary of Akkadian (CDA) (Black, George, and Postgate 200o), as that is the guideline volume used in our text corpus, the Oracc.
} 
mation and examples there. The $C A D$ is not fully searchable, so this part of the research needed to be done by hand. As these proposals are based on the $C A D$, they represent a potentially etic view of the lexemes. This is why the suggestions of this section will be evaluated in light of PMI and Word2vec results in later sections. Tables 7.1-3 highlight syntagmatic relationships. A discussion of the paradigmatic relationships follows each table.

In our close reading of the $C A D$, the aim regarding syntagmatic relationships was to identify the probable text scenarios where the lexeme of interest could appear, the kind of "prototypical" scenarios described above (see Fig. 7.1 and Fig. 7.2). Each such domain has been labeled with a letter and a heading in the table. For example, in Table 7.1 is found category C: Horse as an animal in royal service and war, which includes lexemes that (based on the CAD) tend to occur with "horse" in that particular scenario. This approach has meant that we have excluded words that have semantically a great number of possible contexts (e.g., prepositions) or that appear very rarely with the word of interest. Details of such excluded words are given below in the subsections. Because the analysis is qualitative and the method involves close reading, there is no simple list available for which words were discarded. The endeavor was based on $C A D$ evidence, which of course had to be balanced with the fact that the text material available for the $C A D$ project is not identical with our corpus material (described above in "Theoretical and Methodological Background and Source Material").

We compared the tables that were thus created against a list detailing how many times the words appeared in the text corpus used for this study. Words appearing fewer than ten times were eliminated from the tables. Additionally, one should bear in mind that most compound words (e.g., rabi sisî, lit. "master of horses") are not identified as individual lexemes in our corpus, which is why they did not show up in our analysis. ${ }^{33}$ The lexemes in tables are presented (within each category) in the order of how common they are in our corpus. The number of occurrences is indicated in parentheses after the Akkadian word. Thus, this listing at the same time indicates a rough probability of the individual lexemes appearing in the PMI analyses.

33 Note that approximately 380 compound words are marked with a single lemma in the corpus. 


\section{sisû, horse}

Based on the work of Blois, ${ }^{34}$ it was suggested earlier that in a small sample corpus $^{35}$ the syntagmatic semantic domain of "horse" (Akkadian sisû) could have at least two categories: 1) the WARFARE-domain associates "horse" with "garrison," "chariot," "cavalry," and "attack," whereas 2) the ECONOMIC-domain gives associated words such as "silver," "gold," "to buy," and "gift." Hence, one can suggest that the writers considered the horse mainly as an economic and military resource and not — as in Finland — an important animal in the AGRICULTURE-domain. ${ }^{36}$

This initial analysis was compared to the $c A D,{ }^{37}$ which suggests the following categories: a in general; b for transport; c for riding; $\mathbf{d}$ draft horses; e military contexts; $\mathbf{f}$ booty and tribute; $\mathbf{g}$ as gifts; $\mathbf{h}$ care, pasturing, and training; $\mathbf{i}$ provenance; $\mathbf{j}$ price; $\mathbf{k}$ colors and markings; l qualification by breed, age, and sex; m trappings; and $\mathbf{n}$ personnel. ${ }^{38}$ These categories, which highlight some syntagmatic relationships of "horse," have helped us to compile Table 7.1 below. In order to build syntagmatic categories for sisû (686), "horse," as outlined in the beginning of "Traditional Examples of Semantic Fields," we disregarded some words when creating Table 7.1. For example, nagû, "to bray, bellow, bawl," can in some contexts be translated as "to neigh." Instead, we concentrated on those words that occur in $C A D$ evidence consistently with "horse" in many different $C A D$ categories. The $C A D$ lists approximately fifty lexemes for $C A D$ categories $\mathbf{k}, \mathbf{l}, \mathbf{m}$ and $\mathbf{n}$, but it is impossible to evaluate how commonly these were connected with "horse." We would expect that individual qualifying terms would not show up in quantitative analysis because horses are rarely qualified with any one of these terms. Moreover, these categories were excluded from Table 7.1 because of our focus on finding prototypical scenarios

34 Blois 20o8b.

35 SAA 18 (Reynolds 2003), consisting of 204 texts and dealing mainly with the Babylonian correspondence of King Esarhaddon, served as an example corpus: "horse" appeared in SAA 18 on pages $7,15,56,66,80,112,125,175$, and 197 .

36 Svärd 2012, 58-61; Svärd 2015, 15-19.

$37 \quad C A D$, vol. S, 328-334.

38 The lexeme can also refer to the constellation Horse or to a sea creature (literally "horse of the sea"), but these meanings are rare and not relevant to this discussion. 
TABLE 7.1 Three prototypical contexts for sis $\hat{u}$, “horse" (syntagmatic relationships)

Akkadian lexeme (base form according to English translation (according to the the $C A D$ ) $C D A)$

A. Valuable commodity

$\begin{array}{ll}\text { kaspu }(\mathbf{1}, 686) & \text { silver } \\ \text { maddattu }(209) & \text { payment, obligation, tribute } \\ \text { šulmānu }(\mathbf{2 2}) & \text { greeting gift }\end{array}$

B. Equipment connected with using a horse and taking care of it

$\begin{array}{ll}\text { kurummatu }(317) & \text { food allocation, ration } \\ \text { nīru }(166) & \text { yoke } \\ \text { šǔšānu }\left(5^{6}\right) & \text { horse trainer, groom }\end{array}$

C. Horse as an animal in royal service and war

$\begin{array}{ll}s ̧ \bar{a} b u\left(75^{0}\right) & \text { people, troops } \\ \text { emūqu }(323) & \text { strength, force, military force } \\ \text { narkabtu }(234) & \text { chariot } \\ \text { karāšu }(93) & \text { military camp } \\ \text { rakābum }(91) & \text { to ride } \\ \text { șamādu }(31) & \text { to tie up, yoke, hitch up }\end{array}$

The $C A D$ is not ideal for identifying paradigmatic relationships because its categorizations are mostly based on syntagmatic relationships. However, at least the following comparable animals and designations of different types of horses may be expected to belong to its paradigmatic category: imēru (612), "donkey"; alpum (539), "bull, ox"; kūdanu (114), "mule"; and atānu (31), "she-ass, mare." These lexemes are also likely to show up in an automatic analysis of syntagmatic relationships because they often appear both close to each other and in similar contexts.

\section{qabû, "to speak"}

The verb qabû $(2,353)$, "to speak," is a common verb with a wide semantic range. In Table 7.2, we seek to identify prototypical scenarios where the category can be connected with a fairly specific set of lexemes (based on $C A D$, vol. Q 22-42). There are certain lexemes that seem to often appear together with qabû, but which cannot be connected to any specific semantic domain based 
on the $C A D$ information. Such words include awātu (556), "word, matter, order"; muhhu (3,493), "skull, top, concerning (something)"; magāru (150), "to consent, agree"; pānu (3,233), "front, face" (in the expression ina/ana pāni); aššum (6), "because of, concerning"; umma $(3,303)$, "saying" (a particle introducing direct speech); kiam (102), "so, thus"; and kima $(1,339)$, "like, when, thus." Given the broad semantic nature of the word qabû, we expect that its syntagmatic categories will be difficult to identify. Nonetheless, Table 7.2 presents some possibilities, based on our analysis of the CAD.

TABLE 7.2 Three prototypical contexts for qabû, "to speak" (syntagmatic relationships)

Akkadian lexeme (base form according to the $C D A$ )
English translation (the first meaning

according to the $C D A$ )

A. To state officially (e.g., in court)

$$
\begin{aligned}
& \text { mahru }(\mathbf{1}, \mathbf{1 0 1}) \\
& \text { dìnu }(\mathbf{8 7 3 )} \\
& \text { mimma }(\mathbf{5 3 1}) \\
& \text { dayyānu } \mathbf{( 8 2 )}
\end{aligned}
$$

\section{B. To order}

divine names

šarru (14,389)

šulmu $(\mathbf{1}, 874)$

pû (870)

țūbu (287)

C. To promise (financially)

kaspu $(\mathbf{1}, 686)$

šiqlu (974)

še'u (181) front, before

legal decision, judgment, verdict anything, something, everything judge

any name of a deity

king completeness, well-being (in letters)

mouth

goodness, happiness (in letters)

silver

shekel

barley, grain

The verb qabû was chosen as an object of study nonetheless, because of its potentially interesting paradigmatic relationships. There is reason to suspect that its meaning is not sufficiently conveyed in English expressions. It seems to have specific connotations with authoritative speech, whereas another verb, dabābu (1,163), "to speak," has connotations with informal speech. ${ }^{39}$ Therefore,

39 This is already briefly stated in Svärd $(2015,169)$. According to the $C A D$, vol. D, 4-14, dabābu can mean "to recite" (referring to cultic actions, typically close to divine names or prayer 
the paradigmatic relationships of this word are of great interest. Does dabābu appear in similar contexts as qabû? Or are there other lexemes denoting speech that are closer to qabû than dabābu? A possible candidate for this may be zakāru (209), "to speak."

\section{danānu, "to be strong, powerful"}

Svärd's previous research has explored the concept of "power" in the Neo-Assyrian Empire. ${ }^{40}$ There are many lexemes, usually translated with the English "power" or the German "Macht," but here we are concentrating on one of the most obvious ones, danānu (221), "to be strong, powerful" (cAD vol. D, 83-86), which is connected with the idea of physical strength and force. ${ }^{41}$ Table $7 \cdot 3$ sums up our hypothesis based on the $C A D$.

TABLE 7.3 Five prototypical contexts for danānu, "to be strong"(syntagmatic relationships)

\section{Akkadian lexeme (base form according English translation (at least the first to the $C D A$ ) meaning according to the $C D A$ )}

\section{A. The crown and military}

$\begin{array}{ll}\text { šarru }(14,389) & \text { king } \\ \text { mātu }(4,039) & \text { land, country } \\ \text { nakru }(711) & \text { strange, foreign, enemy } \\ \text { emūqu }(323) & \text { strength, force, army } \\ \text { kabātu }(29) & \text { to be heavy, to be important }\end{array}$

\section{B. To guard}

mașșartu (491)

observation, guard, watch

\section{To make valid, binding}

šì bu $(4,232)$

old, elder, witness

țuppu (537)

tablet, document

terminology) or "to plead in court" (typically close to words related to the derivatives of the verbal root djn, such as diānu in the infinitive "to judge"; dajjānu, "the judge"; dinum, "court case"). It also frequently occurs with the preposition itti (with), because dabābu often refers to reciprocal action.

40 Svärd 2012, 2015.

41 Svärd 2012, 61-65. 
TABLE 7.3 Five prototypical contexts for danānu, "to be strong"(cont.)

Akkadian lexeme (base form according English translation (at least the first to the $C D A$ )

D. To strengthen man-made structures

(canals, foundations, dikes, temple of

baked bricks, structure, reservoir)

bìtu $(5,927)$

house

nāru (46o)

river, canal

dūru (313)

city wall, rampart

igāru (113)

wall

E. To speak/write severely/strongly

šapāru $(2,187)$

awātu (556)

šipțu (31)

to send, send a message

word, matter, order

judgment, verdict

As for paradigmatic relationships, the root of danānu (dnn) has many derivatives, which might show up in similar contexts as danānu. These include dannu (825), "strong, mighty"; dannūtu (50), "strength, power"; and dunnu (16), "power, strength." Other possibilities for paradigmatic companions are derivatives of the root gšr: gašāru (3), "to be strong, powerful"; and gašru (6o), "very strong, powerful." Finally, it seems possible that emūqu (323), "strength, force," could appear in similar contexts as danānu.

\section{Pointwise Mutual Information (PMI)}

PMI is a statistical method used to find collocations and associations between words. It measures the reduction of uncertainty about the occurrence of word $\left(w_{1}\right)$ when word $\left(w_{2}\right)$ is known to be present within a given distance. Thus, we may be able to generate groups of words that are syntagmatically related to each other (see $a b C d e$ axis above in Figures 7.1 and 7.2).

To calculate the PMI scores, we used the Collocations module provided by the Natural Language Toolkit (NLTK), which is the most popular platform for building Natural Language Processing (NLP) programs for Python. ${ }^{42}$ The NLTK

42 Natural Language Toolkit (NLTK): <http://www.nltk.org/> (accessed July 1, 2017). See also Bird, Klein, and Loper (2009). For the Python programming language, see $<$ https://www. python.org/> (accessed July 1, 2017). 
is open-source software, and it includes several powerful and easy-to-use tools, from tokenizers to parsers and visualization.

The method splits the input text into smaller segments of fixed length, called a (collocational) window. The size of the window defines the maximum distance between the elements of the examined bigram consisting of $w_{1}$ and $w_{2}$. Then the frequency of the bigram and the individual frequencies of $w_{1}$ and $w_{2}$ are calculated and converted into probabilities. The PMI score is a logarithmic ratio of the bigram's actual probability to the expected chance that the words of the bigram would co-occur independently. ${ }^{43}$ Mathematically:

$$
\text { PMI }\left(w_{1} ; w_{2}\right)=\log \frac{p\left(w_{1}, w_{2}\right)}{p\left(w_{1}\right) p\left(w_{2}\right)} \quad \begin{array}{ll}
\text { FIGURE } 7.3 \\
\text { Formula for PMI score }
\end{array}
$$

where $\mathrm{p}\left(\mathrm{w}_{1}, \mathrm{w}_{2}\right)$ stands for the joint probability of the words and $\mathrm{p}\left(\mathrm{w}_{1}\right)$ and $p\left(w_{2}\right)$ for their individual probabilities. For example, let us assume that we have a corpus of $1,000,000$ words and we want to measure the PMI score for a bigram consisting of words $\mathrm{w}_{1}$ and $\mathrm{w}_{2}$. Let us also assume that our bigram occurs in the corpus 100 times within a given window, and the separate frequencies for $\mathrm{w}_{1}$ and $\mathrm{w}_{2}$ are 400 and 160 , respectively. By using the formula described above (without yet using the logarithm), we get:

$$
\frac{\frac{100}{1000000}}{\frac{400}{1000000} \times \frac{160}{1000000}} \approx 1562.5
$$

FIGURE 7.4

Example of the formula for the PMI score

If the individual frequencies of $\mathrm{w}_{1}$ and $\mathrm{w}_{2}$ were higher, the expected probability of their independent co-occurrence would increase, and the formula would give us a lower result. If the result drops below 1.0, it indicates that there is a higher probability that the words occur together coincidentally rather than as a true bigram.

By using a logarithm, ${ }^{44}$ we can normalize the outcome in a way that values greater than 1.0 are converted into positive PMI scores and values less than 1.0 into negative PMI scores. With the values given above, we would have a PMI score of 7.35. If the individual frequencies were 4,000 and 1,600, the PMI score would decrease to 2.75 ( $\log 15.63)$, thus indicating a weaker association.

43 Church and Hanks 1989.

44 By common definition, a logarithm (abbreviated "log") is a quantity representing the power to which a fixed number (the base) must be raised to produce a given number. 
As the PMI has a well-known tendency to give high scores for bigrams with low frequency, ${ }^{45}$ several different ways have been proposed to normalize and improve the reliability of the PMI scores. One option is simply to use a high frequency threshold (i.e., to filter out bigrams that occur rarely), but due to the small size of our corpus, this would have caused a loss of potentially interesting data. In order to get better results with low frequency thresholds, we chose to normalize our scores by using Normalized PMI (NPMI). ${ }^{46}$ The NPMI is an extended version of the (PMI) formula, which removes some of the low-frequency bias and gives the score fixed upper and lower boundaries.

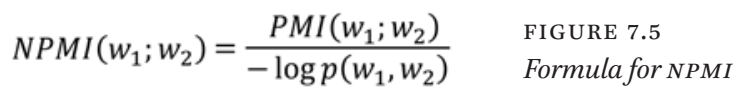

To avoid subjectively choosing one window size and frequency threshold, we used a range of different window sizes from 5 to 25 in increments of 5 with five different frequency thresholds each: 10, 15, 20, 25 and 30. This produced 25 score tables for each word. We chose to pick our final results from the best 20 collocations from each score table. The results were merged by calculating the collocate frequencies $\left(\mathrm{freq}_{\mathrm{c}}\right.$ ) from each score table (i.e., in how many tables they were present at least once) and by using a natural logarithm of this frequency as a weight for their average NPMI scores:

$$
\begin{array}{ll}
S C O R E=N P M I_{\text {avg }} \times \log \text { freq }_{c} & \text { FIGURE 7.6 } \\
& \text { Formula for the final score }
\end{array}
$$

Here the logarithm was used for two purposes: to avoid giving high-frequency collocates too-dominant weights and to filter out all collocates that occurred only once in our score tables (as $\ln 1=0$ ). For example, the following simplified score tables would have given words $a$ and $c$ the freqc of 2 (as they occur in Part I and Part II of Table 7.4) and a logarithmic weight of In $2 \approx .3$; thus, they would have been merged as follows: ${ }^{47}$

\footnotetext{
45 Manning and Schütze 1999; Pantel and Lin 2002.

46 Bouma 2009.

47 Naturally, with only two score tables, filtering out single collocates would not have been justified.
} 
TABLE 7.4 Merging the score tables

\begin{tabular}{lllllll}
\hline $\begin{array}{l}\text { Part I } \\
\text { Collocate }\end{array}$ & NPMI & $\begin{array}{l}\text { Part II } \\
\text { Collocate }\end{array}$ & $\begin{array}{l}\rightarrow \\
\text { NPMI }\end{array}$ & $\begin{array}{l}\text { Merged } \\
\text { Collocate }\end{array}$ & NPMI $_{\text {AVG }}$ & Score \\
\hline$a$ & .3 & $d$ & .3 & $a$ & .25 & .075 \\
$b$ & .2 & $a$ & .2 & $c$ & .15 & .045 \\
$c$ & .1 & $c$ & .2 & $b, d$ & - & 0 \\
\hline
\end{tabular}

The scoring did not only filter out unique collocates, but it also gave a little bias for the high-frequency collocates and bigrams, ${ }^{48}$ of which the latter, as mentioned earlier, are generally considered more reliable. It also allowed some frequent but not so highly scored collocates to appear in our final results. Very similar results could have been achieved by using slightly higher frequency thresholds, but that would have caused more data loss, especially with infrequent words of interest. By using the scoring method described here, the threshold is more adaptive to the words of interest with varying frequencies.

The tables below summarize our final results, which are sorted by the average NPMI scores. The weighted NPMI scores are not shown in the tables, as they were only used for merging the results and filtering out potentially irrelevant collocations. Column "Freq ${ }_{\mathrm{AV}}$ " indicates the average bigram frequency.

TABLE 7.5 NPMI results for the top fifteen suggestions for sisû, "horse"

\begin{tabular}{lllcl}
\hline & Word & Translation & Freq $_{\text {AVG }}$ & NPMI $_{\text {AVG }}$ \\
\hline 1. & Kusaya & Kushite & 91 & .418 \\
2. & pèthallu & riding horse & 82 & .363 \\
3. & parû & mule & 72 & .353 \\
4. & Mesaya & Mesaean & 16 & .349 \\
5. & kūdanu & mule & 109 & .337 \\
6. & udru & Bactrian camel & 20 & .329 \\
7. & sisû & horse & 219 & .293 \\
8. & șēnu & flock & 66 & .293 \\
9. & gammalu & camel & 34 & .288
\end{tabular}

48 The bigram and collocate frequencies are related as averages, and thus giving a penalty for the infrequent collocations in score tables increases the average bigram frequency in the merged table. 
TABLE 7.5 NPMI results for the top fifteen suggestions for sisû, horse (cont.)

\begin{tabular}{lllcl}
\hline & Word & Translation & Freq $_{\text {AVG }}$ & NPMI $_{\text {AVG }}$ \\
\hline 10. & nïbu & naming & 34 & .254 \\
11. & narkabtu & chariot & 57 & .248 \\
12. & nìru & yoke & 43 & .246 \\
13. & gimru & totality & 129 & .229 \\
14. & alpu & ox & 92 & .215 \\
15. & peș̂u & white & 36 & .208 \\
\hline
\end{tabular}

At first glance, the PMI results of Table 7.5 for syntagmatic fields of "horse" do not match up well with the syntagmatic fields proposed by traditional $C A D$ analysis presented in Table 7.1. Table 7.1 presents three hypothetical fields: (A) valuable commodity, (B) equipment relating to horses, and (C) horses in war and royal service. The only two lexemes from Table 7.1 that appear in Table 7.5 are narkabtu (chariot), connecting "horse" to field C, and niru (yoke), connecting "horse" to field B.

We should note, however, that some Table 7.1 words received high NPMI scores but do not show up in Table 7.5 due to their low collocational frequency (see Table 7.4 above). Words that appear in the $C A D$ analysis of the section titled "sisû (horse)" and had a positive NPMI score (but which do not show up in Table 7.5) include: šūšānu(tu), "position of horse-trainer" (.317); rakābu, "to ride" (.206); karāšu, "military camp" (.186); emūqu, "strength, soldier" (.13); and kaspu, "silver" (.003).

In general, the results seem to consist of different draft animals and animals used in expeditions and caravans. Therefore, Table 7.5 seems better at presenting the paradigmatic relationships than syntagmatic relationships. Two of the words in Table 7.5 ("mule" and "ox") appear as hypothetical paradigmatic relationships for "horse" in the section titled "sisû (horse)." The results of PMI also match well the results of Word2vec analysis (see the section "Word2vec"), which specifically aimed to explore the paradigmatic relationships of "horse." This was partially expected in the section titled "sisû, "horse," however, as the lexemes of the paradigmatic group (such as "mule" and "donkey") appear both in similar contexts and close to each other. At least to some degree, this is because many of the occurrences of "horse" and the lexemes of its paradigmatic group appear in lists enumerating, for example, taxes or tribute from foreign lands. This explanation is also supported by the appearance of gimru (total) in the PMI results, as this term is often used to sum up contents in lists. It is also 
supported by the fact that the PMI results exclusively show nouns-a feature that is also typical for lists.

An unexpected result of the PMI analysis is the presence of designations of horses: "Kushite," "Mesaean," and "white." "Kushite" is even the term most often connected to "horse." This is surprising, because there are very many qualifying adjectives for horses. The assumption in the $C A D$ analysis of the section "sisu, 'horse," was that because of the large number of these terms, none of them would show up in PMI analysis. The dominance of "Kushite" might indicate that it is rarely connected to any other word apart from "horse," whereas "white," for example, of course has many other uses.

TABLE 7.6 NPMI results for the top fifteen suggestions for qabû, "to speak"

\begin{tabular}{|c|c|c|c|c|}
\hline & Word & Translation & Freq $_{\text {AVG }}$ & NPMI $_{\text {AVG }}$ \\
\hline 1. & nig̃ak & deed & 26 & .249 \\
\hline 2. & naqbìtu & utterance & 15 & .247 \\
\hline 3 . & teslìtu & appeal & 42 & .204 \\
\hline 4. & bibbulu & flood & 15 & .173 \\
\hline 5 . & ibru & friend & 17 & .159 \\
\hline 6. & $q a b \hat{u}$ & to say & 388 & .138 \\
\hline 7. & apālu & to pay & 32 & .136 \\
\hline 8. & awātu & word & 97 & .128 \\
\hline 9. & zakāru & to speak & 43 & .124 \\
\hline 10. & šâlu & to ask & 69 & .121 \\
\hline 11. & bìtu\&c\&ridûtu ${ }^{\mathrm{a}}$ & "House of Succession" & 23 & .113 \\
\hline 12. & epištu & deed & 30 & .112 \\
\hline 13. & magāru & to consent & 29 & .111 \\
\hline 14. & arazu & supplication & 26 & .111 \\
\hline 15 . & puhru & assembly & 26 & .109 \\
\hline
\end{tabular}

a The symbols "\&\&" indicate a compound word.

In the case of qabu (and verbs in general), using only the average NPMI scores would have produced very obscure results, ${ }^{49}$ and even with the weighted

49 For example, the top ten results contain such words as bahru, "boiling hot one" (.252); anam, "what" (.205); tartugallu, "hen," from KUR DAR.LUGAL.MEŠMUŠsN, "land of roosters" (.177); and sittūtu, "those remaining" (.174). 
scores the results are open to some interpretation. Somewhat better results can be achieved by using very narrow collocational windows:

TABLE 7.7 NPMI results for the top fifteen suggestions for qabû, "to speak," when using a window size of 5

\begin{tabular}{|c|c|c|c|c|}
\hline & Word & Translation & Freq $_{\text {AVG }}$ & NPMI $_{\text {AVG }}$ \\
\hline 1. & teslìtu & appeal & 27 & .275 \\
\hline 2. & zakāru & to speak & 27 & .192 \\
\hline 3. & awātu & word & 38 & .143 \\
\hline 4. & šâlu & to ask & 26 & .138 \\
\hline 5 . & šiptu & incantation & 20 & .117 \\
\hline 6. & $p \hat{u}$ & mouth & 47 & .116 \\
\hline 7. & mahāru & face & 20 & .106 \\
\hline 8. & țēmu & (fore)thought & 30 & .104 \\
\hline 9. & alāku & to go & 136 & .098 \\
\hline 10. & šarru & king & 577 & .095 \\
\hline 11. & šemû & to hear & 28 & .094 \\
\hline 12. & $q a b \hat{u}$ & to say & 73 & .093 \\
\hline 13. & ере̄šu & to do & 75 & .082 \\
\hline 14. & bèlu & lord & 467 & .081 \\
\hline 15. & balāṭu & life & 22 & .078 \\
\hline
\end{tabular}

We then compared Tables 7.6 and 7.7 to the hypotheses formulated with the help of the $C A D$ in the section titled "qabu, 'to speak." As expected, in this section the syntagmatic relationships of qabû were difficult to establish with PMI, due to its wide semantic range. The results for words that appear close to qab $\hat{u}$ in both tables include awātu (word, matter) and, in Table 7.6, magāru (to consent). These were expected to occur with qabû, but they cannot be connected to any clear semantic domain or prototypical scenario.

The word qabû, "to speak," could have hypothetically appeared in three prototypical scenarios: (A) to state officially (e.g., in court), (B) to order, and (C) to promise (financially). The only one of these that has a connection to PMI results is B, with the words pî and šarru appearing in Table 7.7. We should note, however, that when looking at simple average NPMI scores, the following additional words from Table 7.2 appeared in our results: dayyānu, "judge" (.064); šulmu, "well-being" (.035); and mahru, "front" (.o12). 
In these results, we also note a verb associated with speaking: šemû, "to hear." We can also see the typical participants in Mesopotamian texts involving speaking, lords (bēlu) and kings (šarru), who typically order someone to do (epēšu) something, such as going (alāku) somewhere, and who are usually appealed to (teslitu) by their subjects. Finally, as with "horse" above, the paradigmatic relationships seem to be represented here, or at least the appearance of zakāru (to speak) suggests it.

TABLE 7.8 NPMI results for the top fifteen suggestions for danānu, "to be strong"

\begin{tabular}{|c|c|c|c|c|}
\hline & Word & Translation & Freq $_{\text {AVG }}$ & NPMI $_{\text {AVG }}$ \\
\hline 1. & šupku & foundation & 13 & .387 \\
\hline 2. & birtūtu & function of fort & 11 & .382 \\
\hline 3. & enēšu & to be(come) weak & 14 & .303 \\
\hline 4. & takālu & to trust & 15 & .277 \\
\hline 5. & pïlu & limestone & 22 & .273 \\
\hline 6. & $e w \hat{u}$ & to become & 18 & .271 \\
\hline 7. & epištu & deed & 16 & $.25^{1}$ \\
\hline 8. & temmēnu & foundation & 16 & .251 \\
\hline 9. & lìtu & victory & 12 & .241 \\
\hline 10. & mušarû & (royal) inscription & 14 & .22 \\
\hline 11. & šațāru & to write & 28 & .215 \\
\hline 12. & bēlūtu & rule & 23 & .188 \\
\hline 13. & dūru & city wall & 16 & .188 \\
\hline 14. & șēru & back & 24 & .177 \\
\hline 15 . & nišu & people & 29 & .135 \\
\hline
\end{tabular}

Finally, we compared Table 7.8 with Table 7.3 (in the section titled "danānu, 'to be strong, powerful"). The word danānu, "to be strong," was expected to appear in five syntagmatic domains: (A) the crown and military, (B) to guard, (C) to make valid, (D) to strengthen man-made structures, and (E) to speak or write strongly.

Interestingly, the results of Table 7.8 seem to be connected with three of the suggested syntagmatic fields of Table 7.3: namely, A, D and E. Only one of the lexemes in Table 7.3 appears explicitly—namely, "city wall"—but three other terms in Table 7.8 fit the bill for syntagmatic field D: namely, the two terms translated as "foundation" and "limestone." Regarding the proposed field E, although we do not find țuppu, "tablet"; or šapāru, "to send," in our results, some 
words with similar meanings such as musarû, "(royal) inscription"; and sațāru, "to write," are present instead in Table 7.8. Finally, regarding A, there are several terms in Table 7.8 that are connected with crown and military: "function as fort," "victory," "(royal) inscription," and "to rule."

As far as danānu is concerned, the following expected words of syntagmatic relationships (see "danānu [to be strong, powerful]") had positive NPMI values: dūru, "city wall" (.188); emūqu, "soldier, strength" (.168); mașșartu, "to guard, watch" (.139); nakru, "enemy" (.109); mātu, "land" (.o97); and šarru, "king" (.oog). The low NPMI score of šarru can be explained by the word's very high individual frequency and by the fact that Oracc does not lemmatize dannu as danānu, which prevents its most obvious bigram šarru dannu, "a mighty king," from showing up in our results. In general, several collocates seem to come from different texts describing the strengthening of structures or conquering new lands. The antonym enēšu can be explained by formulas such as: "I strengthened it, so that it would not become weak." For paradigmatic relationships, as outlined based on the $C A D$ in "danānu, "to be strong, powerful," there is little evidence in the PMI results.

\section{Word2vec}

Whereas PMI might suggest syntagmatic semantic fields, Word2vec should be highlighting paradigmatic relationships of the words of interest (see Figs. 7.1 and 7.2 above). Word2vec is one of the methods in the field of Natural Language Processing (NLP) that can map vocabulary units to vectors of real numbers. ${ }^{50}$ Word2vec models plot a unique vector in space for each lexeme in the corpus. The models align these vectors in such a way that words appearing in similar linguistic contexts cluster near each other, thus giving us information on possible paradigmatic groups of words. The text to be analyzed is converted via a hidden layer to a multidimensional output vector that gives the probability distribution of the words. ${ }^{51}$ Word2vec uses so-called ANNs to predict word co-occurrence.

Word2vec was developed by Tomas Mikolov and his team in $2013,^{52}$ but the underlying idea that "a word is characterized by the company it keeps" was popularized as early as the 1950 s by John R. Firth, ${ }^{53}$ who in turn was influenced

\footnotetext{
50 Mikolov et al. 2013a; Mikolov, Yih, and Zweig 2013.

$5^{1} \quad$ Mikolov, Yih, and Zweig 2013.

52 Mikolov et al. 2013a; Mikolov, Yih, and Zweig 2013.

53 Firth 1957.
} 
by early anthropological studies. The ideas presented by Firth definitely have the essence of other branches of the humanities emphasizing the importance of context in any endeavor of researching culture and language.$^{54}$ Word2vec is an open-source toolkit for producing word vectors and querying semantic relationships between words. 55

There are two alternative models one can choose from when using Word2vec: the Continuous Skip-gram model and the Continuous Bag-of-Words (своw) model. ${ }^{56}$ When training the data, the Continuous Skip-gram model predicts the words that may appear near the target word, ${ }^{57}$ whereas the свош model uses the various words that can appear in the same context as the target word for the prediction. A detailed explanation of these methods is beyond the scope of this paper.

The last stage of converting the hidden layer into the output vector is computationally expensive and time-consuming. Hence, there are several ways of approximating this step. In Word2vec, one can choose between two such methods for creating the output vector: hierarchical softmax and negative sampling. In hierarchical softmax, the values for the words are stored in such a way that not all of the words have to be processed when calculating the probability of a word. Negative sampling is a procedure in which only a small part of the model's weights is updated. According to Mikolov, ${ }^{58}$ these methods not only speed up the training, but they also improve the accuracy of the prediction. When looking for words appearing together with other words, one is not interested in the most frequent words, such as "the" in English. According to Mikolov, ${ }^{59}$ a method called subsampling efficiently reduces the effect these very frequent words have on the training. In Word2vec, it is also possible to

54 Firth 1957. See also the discussion on emic approaches above in "Emic Approach and Linguistic Departure Points."

Mikolov et al. 2013a. The original link is not working anymore, but many copies of the toolkit can still be found on Github. The kit we used was downloaded on November 14, 2016 from <https://github.com/dav/word2vec > (accessed July 7, 2017).

$5^{6}$ Mikolov et al. 2013a. Continuous Bag-of-Words: one of two alternative models one can choose from when training data in Word2vec. While training, the своw model uses the various words that can appear in the same context as the target words for the prediction of semantic relationships. For further explanation of the bag-of-words model, see in this volume, Monroe, 270-272.

57 Continuous Skip-gram model: one of two alternative models one can choose from when training data in Word2vec. While training, the Continuous Skip-gram model predicts the words that may appear near the target word.

$5^{8} \quad$ Mikolov et al. 2013a.

59 Mikolov et al. 2013a. 
choose the window size of the context in which the words are considered. For example, a window size of 5 would consider five words before and five after a word. However, in practice, Word2vec picks a random number $R$ within the range chosen ( 1 to 5 in the previous example), and it considers $R$ words on each side of the target word. ${ }^{60}$

Depending on the values chosen for the different parameters used with Word2vec, the resulting multidimensional vectors differ from each other. Furthermore, because of randomness and subsampling, the results also change from one computing session to another. Thus, the results presented here are just an approximation. We experimented with different parameters and noticed that the dimension of the vector has a smaller effect on the results than the threshold used for subsampling. According to Mikolov, ${ }^{61}$ a typical threshold for this would be .oooo1. That would mean that words with a relative frequency greater than this might be disregarded. As our texts have only 12,067 different word types, using a small threshold did not give good results. In the tests conducted by Mikolov and his team, ${ }^{62}$ negative sampling worked better than hierarchical softmax. Therefore, we used negative sampling. Although values of $5^{-20}$ are recommended by Mikolov and his team for negative sampling in small training sets and $2-5$ in large ones, we found that using too many samples for training did not work well with our data. We used the following parameters in our analysis: vector dimensionality 200, negative sampling with 4 samples, subsampling with threshold .01, and window size 7 . We ran the script provided in the Word2vec toolkit with these parameters 500 times, using the cвоw model architecture and our own text file. After each run, the word vector produced by Word2vec was discarded, as we wanted the tool to create a new one with different random samples. From each run, we queried for the $5^{0}$ words that were semantically closest to each of the test words (sis $\hat{u}, q a b \hat{u}$, and danānu). After the runs, we scored the words received from Word2vec, so that the closest match to a test word was scored 1 and the furthest 50 . For every run a word that was counted at least once not appearing in the list, a penalty of 51 points was scored. The average score was then counted for each word. In this way, we ended up with a list of words that were closest to the target word in all 500 runs. Ten of the closest words for each of the target words sis $\hat{u}$, qab $\hat{u}$, and danānu can be seen in Tables 7.9-11. The numbers after the words indicate the average placement that a particular word appeared in during the 500 runs exe-

\footnotetext{
6o Mikolov et al. 2013b; Levy and Goldberg 2014.

$61 \quad$ Mikolov et al. 2013a.

62 Mikolov et al. 2013a.
} 
cuted, thus reflecting how high in the list of words returned by Word2vec that word was on average.

TABLE 7.9 Word2vec results for the top ten suggestions for sisû, "horse"

\begin{tabular}{lll}
\hline Word & Score & Translation \\
\hline kūdanu & 1.3 & mule \\
șumbu & 1.7 & wheel \\
parû & $3 \cdot 3$ & mule \\
gammalu & 5.6 & camel \\
udru & 5.8 & Bactrian camel \\
narkabtu & $7 \cdot 1$ & chariot \\
șēnu & $7 \cdot 9$ & flock \\
namrāṣu & 8.6 & hardship \\
Kusaya & $9 \cdot 3$ & Kushite \\
mānu & $9 \cdot 7$ & counting \\
\end{tabular}

As expected, the Word2vec analysis for "horse" found the words that appear in similar contexts as "horse" - that is to say, potential paradigmatic relationships (see "Emic Approach and Linguistic Departure Points"). Lexeme to lexeme, the results are not an exact match, and only 1 out of 4 suggestions (kudānu) made its way onto the Word2vec list. However, most of the other words appearing in the Word2vec list clearly belong to the same conceptual domain. In addition to kudānu, "mule," which was suggested in traditional analysis, we find two words for "camel" and a different word for "mule," for example. From the words suggested by the traditional method, we find alpu, "ox," in position 11, with an average score of 9.7, and imēru, "donkey," in position 12, with a score of 15.9 . The word atānu can be found in shared 179th position from a total of 372 words. The average scores (1.3-9.7) of the ten closest words to sisu, "horse," reveal that these words appeared very high on the list on almost every run.

A little surprisingly, we find a clear indication here of the semantic domain of war as well, with "chariot" and "wheel" being mentioned. These match the proposed syntagmatic group for "horse" (see "sisû, 'horse"), but we would have expected to find them surrounding "horse" in prototypical scenarios in the PMI analysis (see "Pointwise Mutual Information"), not as words that appear in a similar environment. Such results could suggest that separating syntagmatic and paradigmatic relationships statistically might be more difficult than we anticipated. 
TABLE 7.10 Word2vec results for the top ten suggestions for qabû, "speak"

\begin{tabular}{lll}
\hline Word & Score & Translation \\
\hline magāru & 2.7 & to consent \\
awātu & 2.9 & word \\
wadûu & 2.9 & to know \\
hasāsu & 5.2 & to be(come) conscious \\
kamsu & $9 \cdot 3$ & gathered \\
šâlu & 12.3 & to ask \\
šipirtu & 14.9 & message \\
mītu & 19.9 & dead \\
hibiltu & 21.2 & wrongdoing \\
abāku & 21.7 & to overturn \\
\end{tabular}

The results of Word2vec for qabû were generally quite similar to the PMI results. Interestingly, the Word2vec analysis did not show any other words that are traditionally translated as "to speak." This might support the idea that each of the verbs related to speaking (e.g., dabābu, zakāru, and perhaps awû) has a semantic domain of its own, as hypothesized in "sisû, "horse" above.

The average scores $(2.7-21.7)$ in the lists of words given by Word2vec for qabû are quite a lot higher than for sisû, which might indicate that there is a larger variety of words appearing close to the word qabû than to sisû. Outside the list of the top ten words found, the word dabäbu, "to speak," is on the list in the shared 91st place, with the average score of 49.1. The word $a w \hat{u}$, "to speak," was present on only two runs. The word zakäru, however, is not on the list of closest words to qabû; this is interesting, as it does show up in Tables 7.6 and 7.7 of the PMI results that presumably suggest syntagmatic categories.

The paradigmatic relationships suggested by Word2vec for danānu bear some similarity to the proposals based on the $C A D$ (see "danānu, 'to be strong, powerful"'). Regarding words suggested by the analysis of the $C A D$, one finds the word gašru in the first position in Table 7.11. Words with the root $d n n$ are present in the form of dandannu, "all-powerful."63 The words gašăru and emūqu were not found in the analysis. When we compare this to the syntagmatic proposals of PMI, we find that the lexemes are fairly dissimilar, as there is only one word that appears directly in both: lìtu, "victory."

63 The word dunnu appeared in only two runs. 
TABLE 7.11 Word2vec results for the top ten suggestions for danānu (dnn), "to be strong, powerful"

\begin{tabular}{lll}
\hline Word & Score & Translation \\
\hline gašru & 5.2 & strong \\
šeriktu & 13.3 & present \\
ilūtu & 14.9 & divinity \\
lìtu & 16.6 & victory \\
qurdu & 17.6 & warriorhood \\
liptu & 18.4 & undertaking \\
dandannu & 19.3 & all-powerful \\
rāimu & 19.5 & loving \\
agîu & 21.4 & crown \\
rimìtu & 21.4 & residence \\
\end{tabular}

The average scores $(5.2-21.4)$ for the words returned for danānu, "to be strong," are even higher than the ones for qabu. It is difficult at this point in the research to say whether this is due to the fact that danānu was a less frequently used word than the other two. It appears in the test corpus only 221 times, whereas sisû appears 686 times and qabû appears 2,353 times. This could also be the reason why the results of Word2vec analysis for danānu were more varied than for sisû or qabû.

\section{Discussions and Future Prospects}

To sum up the results, our aim was to examine the usefulness of the PMI and Word2vec methods for constructing syntagmatic and paradigmatic semantic fields for Akkadian by using sisû, "horse"; qabû, "to speak"; and danānu, "to be strong, powerful," as test cases. After a discussion of theory, methodology, and our sources ("Theoretical and Methodological Background and Source Material" and "Emic Approach and Linguistic Departure Points"), we suggested both paradigmatic and syntagmatic semantic domains of Akkadian based on the current semantic knowledge of Assyriology ("Traditional Examples of Semantic Fields"). These suggestions were compared with the results of PMI analysis for syntagmatic relationships ("Pointwise Mutual Information") and Word2vec for potential paradigmatic relationships ("Word2vec"). These first results of our research group offer some promise that quantitative data on the 
connections between individual lexemes can indeed help Assyriologists understand the ancient contexts better. At the very least, the detailed suggestions for semantic domains generated with the help of PMI and Word2vec enable Assyriologists to look in the right direction. Any detailed Assyriological work on any specific semantic field will of course need to be backed up with solid philological work on the actual word occurrences in primary sources. We see the importance of the current contribution not so much in the light that it can

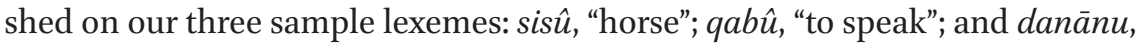
"to be strong, powerful." Rather, we see its main value in showing that methods like PMI and Word2vec can be profitably used to help define the semantics of individual words. For language-technological work, the interconnections between results gleaned with PMI and results from Word2vec suggest that automatically differentiating between paradigmatic and syntagmatic sematic fields will need more research.

As for the future plans of our research group, we intend to proceed with a diachronic approach and also analyze our results by text genre. Such comparisons may be able to illuminate possible changes in semantic domains. The written history of Mesopotamia in the Akkadian language covers roughly the years 2300-300 BCE. The dialects of Akkadian during these centuries have been studied, of course, but not from the perspectives suggested here: semantic domains and long-term continuities and changes in these domains. Thus, an interesting and important research project would be to do similar analyses as here, but only for parts of our corpus. As we are dealing with several different dialects of Akkadian and several different text genres, it would be interesting to see what kinds of differences there are between them and how such differences might be explained from the perspective of the history of the Akkadian language. For example, the possibility of identifying language contacts and influences from other languages is intriguing.

For archaeology, understanding the paradigmatic relationships better would help interpretations of the material records. From a methodological perspective, understanding the methods of word sense induction (paradigmatic and syntagmatic concepts) and how they can be applied could be a useful tool when developing semiotic models for interpreting archaeological records.

Finally, further results might also offer valuable input for a comparison of languages: do the contextual semantic frames of Akkadian match those constructed for modern languages or for biblical Hebrew? Furthermore, they might shed light on the questions: how much of the contextual semantic frames stay the same from language to language and to what degree are languages really unique cognitive constructions? 
On a practical level, we might need to take better into account the specific challenges presented by the corpus. Many of our texts are damaged or only fragments of the original texts. Ideally, we would find a way to model the missing pieces of information in such a way that the methods designed for complete texts or sentences will still be able to provide meaningful results. However, at least for the results presented here, our rather simple solution of excluding damaged parts from the analysis seems to have worked, at least to some extent.

Another challenge is that many of the culturally significant texts, such as the Epic of Gilgameš or the royal inscriptions, were found in several partially preserved copies. The presence of duplicate or near-duplicate texts could be considered in a more nuanced way when using statistical methods. Members of our team have encountered the same problem when harvesting Finno-Ugric texts from the internet. ${ }^{64}$ For this paper, we have simply treated the composite master text as "the text," without taking into account manuscript variants.

The amount of information created by looking for semantic fields with the methods of computational linguistics is immense. That is why one of the longterm goals of our research group is to create a web interface for browsing the different points of view created by varying the time, type, quality, etc., of the texts being analyzed. As filters, we may be able to use some of the metadata that accompanies the texts in the corpus (on the corpus metadata, see the discussion in "Theoretical and Methodological Background and Source Material"). Such an interface could perhaps also be used for other, relatively small text corpora, especially those in extinct and endangered languages.

In terms of visualizing the information on semantic domains, the semantic relationships between lexemes can also be conceptualized as networks, with nodes and edges. Thus, the changing relationships between semantic fields throughout the timeframes could be demonstrated via Gephi or some other software designed to visualize and analyze large networks. ${ }^{65}$

These are some of the team's first results. As we have years of work ahead of us, we look forward to continuing to develop these approaches further. The results will be open to the research community, and all the software tools built in the project will be published as open-source. In the future, the research will benefit from additional sources, as texts are continuously being added to Oracc.

64 Jauhiainen, Jauhiainen, and Lindén 2015.

65 Gephi: <https://gephi.org/about/> (accessed July 1, 2017). See also Pagé-Perron, 209. 


\section{References}

Arens, William, and Ivan Karp. 1989. "Introduction." In Creativity of Power: Cosmology and Action in African Societies, edited by William Arens and Ivan Karp, xi-xxix. Washington, DC: Smithsonian Institution.

Baroni, Marco, Georgiana Dinu, and Germán Kruszewski. 2014. “Don’t Count, Predict! A Systematic Comparison of Context-Counting vs. Context-Predicting Semantic Vectors." In Proceedings of the 52nd AnnualMeeting of the Association for Computational Linguistics, 22-27 June 2014, Baltimore, Maryland, USA, vol. 1, 238-247. Stroudsburg, PA: Association for Computational Linguistics.

Barr, James. 1961. The Semantics of Biblical Language. London: Oxford University Press. Bird, Steven, Ewan Klein, and Edward Loper. 2009. Natural Language Processing with Python. Sebastopol, CA: O'Reilly Media Inc.

Black, Jeremy, Andrew George, and John Nicholas Postgate. 2000. A Concise Dictionary of Akkadian, 2nd corrected ed. SANTAG 5. Wiesbaden: Harrassowitz.

Blois, Reinier de. 2008a. "New Tools and Methodologies for Biblical Lexicography." In Foundations for Syriac Lexicography III: Colloquia of the International Syriac Language Project, edited by Janet Dyk and Wido van Peursen, 203-216. Perspectives on Syriac Linguistics 4. Piscataway, NJ: Gorgias Press.

Blois, Reinier de. 2008b. "Semantic Domains for Biblical Greek: Louw and Nida's Framework Evaluated from a Cognitive Perspective." In Foundations for Syriac Lexicography III: Colloquia of the International Syriac Language Project, edited by Janet Dyk and Wido van Peursen, 265-278. Perspectives on Syriac Linguistics 4. Piscataway, NJ: Gorgias Press.

Bouma, Gerlof. 2009. "Normalized (Pointwise) Mutual Information in Collocation Extraction." In Von der Form zur Bedeutung: Texte automatisch verarbeiten / From Form to Meaning: Processing Texts Automatically, Proceedings of the Biennial GSCL Conference, edited by Christian Chiarcos, Richard Eckart de Castilho, and Manfred Stede, 31-40. Tübingen: Narr.

Church, Kenneth Ward, and Patrick Hanks. 1989. "Word Association Norms, Mutual Information, and Lexicography." In Proceedings of the 27th Annual Meeting of the Association for Computational Linguistics, 26-29 June 1989, Vancouver, British Columbia, Canada, 76-83. Stroudsburg, PA: Association for Computational Linguistics.

Dirven, René, and Marjolijn Verspoor, eds. 2004. Cognitive Exploration of Language and Linguistics. Amsterdam:J. Benjamins.

Eriksen, Thomas H. 2010. Small Places, Large Issues: An Introduction to Social and Cultural Anthropology, 3rd ed. Anthropology, Culture and Society. London: Pluto Press.

Firth, John R. 1957. Studies in Linguistic Analysis. Oxford: Blackwell.

Fleisch, Axel. 2007. "How Cognitive Semantics Relate to Comparative Linguistics: A Case Study from Nguni." In Viva Africa 2007: Proceedings of the 2nd International Conference 
on African Studies, edited by Tomáš Machalík and Jan Záhořík, 39-53. Pilsen: University of West Bohemia.

Geeraerts, Dirk. 2010. Theories of Lexical Semantics. Oxford: Oxford University Press.

Jauhiainen, Heidi, Tommi Jauhiainen, and Krister Lindén. 2015. “The Finno-Ugric Languages and The Internet Project." In First International Workshop on Computational Linguistics for Uralic Languages, edited by Tommi A. Pirinen, Francis M. Tyers, and Trond Trosterud, 87-98. Septentrio Conference Series 2. Tromsø, Norway: Septentrio Academic Publishing.

Kataja, Laura and Kimmo Koskenniemi. 1988. "Finite-state Description of Semitic Morphology: A Case Study of Ancient Accadian." In coling Bdapest: Proceedings of the 12th International Conference on Computational Linguistics, Budapest 22-27.08.1988, edited by Dénes Vargha, 313-315. Budapest:John von Neumann Society for Computing Sciences.

Lakoff, George. 1987. Women, Fire, and Dangerous Things: What Categories Reveal about the Mind. Chicago: University of Chicago Press.

Landsberger, Benno. 1976. The Conceptual Autonomy of the Babylonian World. Translated by Benjamin Foster, Thorkild Jacobsen, and Heinrich von Siebenthal. Monographs on the Ancient Near East 1 (4). Malibu, CA: Undena. Originally published in 1926 as Die Eigenbegrifflichkeit der babylonischen Welt:Ein Vortrag. Sonderausdruck aus Islamica 2 (3), 355-372. Leipzig: Asia Major.

Levinson, Stephen C. 2003. Space in Language and Cognition: Explorations in Cognitive Diversity. Language, Culture, and Cognition 5. Cambridge: Cambridge University Press.

Levy, Omer and Yoav Goldberg. 2014. "Dependency-Based Word Embeddings." In Proceedings of the 52nd Annual Meeting of the Association for Computational Linguistics, 22-27 June 2014, Baltimore, Maryland, USA, vol.1, 302-308. Stroudsburg, PA: Association for Computational Linguistics.

Manning Christopher D., and Hinrich Schütze. 1999. Foundations of Statistical Natural Language Processing. Cambridge, MA: MIT Press.

Mikolov, Thomas, Ilya Sutskever, Kai Chen, Greg Corrado, and Jeffrey Dean. 2013a. "Distributed Representations of Words and Phrases and their Compositionality." In Advances in Neural Information Processing Systems 26 (NIPS 2013): 27th Annual Conference on Neural Information Processing Systems 2013; December 5 - 10, Lake Tahoe, Nevada, edited by Christopher J.C. Burges, Léon Bottou, Max Welling, Zoubin Ghahramani, and Kilian Q. Weinberger. Red Hook, NY: Curran. <https://arxiv.org/ abs/1310.4546>.

Mikolov, Thomas, Kai Chen, Greg Corrado, and Jeffrey Dean. 2013b. "Efficient Estimation of Word Representations in Vector Space." Last modified September 7, 2013. < https:// arxiv.org/abs/1301.3781>. 
Mikolov, Thomas, Wen-tau Yih, and Geoffrey Zweig. 2013. "Linguistic Regularities in Continuous Space Word Representations." In Proceedings of NAACL-HLT 2013, Atlanta, Georgia, USA, 9-14June 2013, 746-751. Stroudsburg, PA: Association for Computational Linguistics.

Nurmikko-Fuller, Terhi. 2016. "Publishing Sumerian Literature on the Semantic Web." Paper presented at the ASOR annual meeting, San Antonio, TX.

Pantel, Patrick, and Dekang Lin. 2002. "Discovering Word Senses from Text." In Proceedings of the SIGKDD Conference on Knowledge Discovery and Data Mining, New York, NY, USA-August 24-27, 2014, edited by Sofus Macskassy and Claudia Perlich, 613-619. New York: Association for Computing Machinery.

Reynolds, Frances. 2003. The Babylonian Correspondence of Esarhaddon and Letters to Assurbanipal and Sin-šarru-iškun from Northern and Central Babylonia. SAA 18. Helsinki: Helsinki University Press.

Roth, Martha T., ed. 1956-2011. The Assyrian Dictionary of the Oriental Institute of the University of Chicago ( $C A D), 26$ volumes. Chicago: The Oriental Institute of the University of Chicago.

Streck, Michael P. 2010. Großes Fach Altorientalistik: Der Umfang des keilschriftlichen Textkorpus. MDOG 142: 35-58.

Svärd, Saana. 2012. "Power and Women in the Neo-Assyrian Palaces." PhD diss., University of Helsinki.

Svärd, Saana. 2015. Women and Power in Neo-Assyrian Palaces. SAAS 23. Helsinki: NeoAssyrian Text Corpus Project.

Tyndall, Stephen. 2012. "Towards Automatically Assembling Hittite-Language Cuneiform Tablet Fragments into Larger Texts." In Proceedings of the 5oth Annual Meeting of the Association for Computational Linguistics, July 8-14, 2012, Jeju Island, Korea, vol. 2, 243-247. Stroudsburg, PA: Association for Computaitonal Linguistics.

w3C. "w3C Semantic Web Activity." Last modified December 11, 2013. <https://www. w3.org/2001/sw/>.

Waerzeggers, Caroline. Forthcoming. "Social Network Analysis of Cuneiform Archives: A New Approach." In Proceedings of the Second START Conference in Vienna, edited by Heather D. Baker and Michael Jursa.

Wolde, Ellen van. 2009. Reframing Biblical Studies: When Language and Text Meet Culture, Cognition, and Context. Winona Lake, IN: Eisenbrauns. 